\title{
Nonrigid Registration of Monomodal MRI Using Linear Viscoelastic Model
}

\author{
Jian Yang, ${ }^{1}$ Yang Chen, ${ }^{2}$ Jingfan Fan, ${ }^{1}$ and Songyuan Tang ${ }^{1}$ \\ ${ }^{1}$ Beijing Engineering Research Center of Mixed Reality and Advanced Display, School of Optics and Electronics, \\ Beijing Institute of Technology, Beijing 100081, China \\ ${ }^{2}$ Laboratory of Image Science and Technology, Southeast University, Nanjing 210096, China \\ Correspondence should be addressed to Songyuan Tang; sytang@bit.edu.cn
}

Received 14 January 2014; Accepted 22 March 2014; Published 22 April 2014

Academic Editor: Zhiwu Liao

Copyright (C) 2014 Jian Yang et al. This is an open access article distributed under the Creative Commons Attribution License, which permits unrestricted use, distribution, and reproduction in any medium, provided the original work is properly cited.

\begin{abstract}
This paper describes a method for nonrigid registration of monomodal MRI based on physical laws. The proposed method assumes that the properties of image deformations are like those of viscoelastic matter, which exhibits the properties of both an elastic solid and a viscous fluid. Therefore, the deformation fields of the deformed image are constrained by both sets of properties. After global registration, the local shape variations are assumed to have the properties of the Maxwell model of linear viscoelasticity, and the deformation fields are constrained by the corresponding partial differential equations. To speed up the registration, an adaptive force is introduced according to the maximum displacement of each iteration. Both synthetic datasets and real datasets are used to evaluate the proposed method. We compare the results of the linear viscoelastic model with those of the fluid model on the basis of both the standard and adaptive forces. The results demonstrate that the adaptive force increases in both models and that the linear viscoelastic model improves the registration accuracy.
\end{abstract}

\section{Introduction}

A current major topic in computational neuroanatomy is the development of nonrigid image registration algorithms. Nonrigid image registration has many potential applications. It can be applied to building atlases, segmentation, quantifying local morphological differences, comparing the variance of different population, and detecting pathological changes [1-4]. Various methods have been developed to deal with nonrigid image registration. The methods are usually classified into two categories: feature-based and intensity-based [5]. The former first needs to build a geometric model and identify a number of anatomic characters in the model. These characters include point landmarks, curves, and surfaces [68]. The anatomical characters are then parameterized. The aim of the registration is to find the optimal combinations of the model parameters. These methods are critically dependent on feature extraction quality. However, the anatomical structures are complex, making it difficult to extract them accurately. Usually, human interaction is required during registration, thereby making the process inconvenient and time consuming. The intensity-based method is used to match regional intensity patterns based on mathematical or statistical criteria [9-13]. This method reduces the need for direct feature extraction or segmentation, can be automatic, and can obtain satisfactory results and is thus widely used. Fluid registration uses physics models and assumes that the deformation obeys fluid mechanics laws. These methods allow flexible deformation with large freedom and are used in many applications.

In the early 1980s, the elastic model was proposed as a means to match images [14]. Brain images are modeled as an elastic solid and the deformations are calculated from elastic mechanic equations $[15,16]$. However, this model is only suitable for small deformations. To address this problem, the properties of brain images were assumed to be like those of viscous fluid, and the viscous fluid model was proposed [17], where the deformations are driven by forces that are equal to the gradient of the sum of squared intensity difference (SSD) metrics. The orientation and magnitude of deformation fields are computed using the fluid-dynamical Navier-Stokes equation. This method allows large deformations and serious 


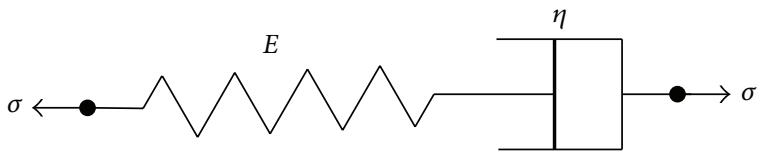

FIGURE 1: Maxwell model is made of springs $(E)$ and dashpots $(\eta)$ in series, and the forces acting on two parts are equal.

localized distortions, but with increased likelihood of misregistration [18]. The Navier-Stokes equation is solved by means of the relaxation method and requires much time. To reduce the computation cost, Bro-Nielson proposed a convolution filter method to solve the equation quickly [19]. However, Wollny et al. [20] obtained unsatisfactory results when using a small filter width in the convolution filter methods. However, if the filter width is large, the computational costs are not more advantageous than when using iterative methods. Thus the relaxation method is currently the best method. The brain images are also modeled as diffusions [21-23] and have been shown to be similar to the method proposed by Bro-Nielsen et al. [19].

In this study, we try to use the properties of both elastic solids and viscous fluids to register images. Linear viscoelastic matter has these properties. The deformation properties of brain images are assumed to be similar to those of viscoelastic matter and obey viscoelastic laws [24, 25]. As the Maxwell model has the abilities to describe linear viscoelastic deformation [26], this study hence utilizes the Maxwell model to represent and capture large deformation of the brain images. When a force acts on the Maxwell model, the motion of the fluid component relaxes over time, allowing large displacements. The deformation fields are constrained by both elastic and fluid components. To speed up the algorithm, an adaptive force is introduced. Given our aim of monomodal anatomic image registration, the SSD is used as a similarity metric in the registration. Both synthetic and real images are used to demonstrate the performance of the proposed method. The performances of both models (Maxwell and fluid) with both forces (standard and adaptive) are compared with each other. The fluid model with adaptive forces (FMAF) has the fastest registration speed, the Maxwell model with adaptive force (MMAF) is the second, the Maxwell model with standard force (MMSF) is the third, and the fluid model with standard force (FMSF) is the slowest. The ranking of registration accuracy from high to low is as follows: MMAF, MMSF, FMAF, and FMSF.

\section{Materials and Methods}

2.1. Maxwell Model. The Maxwell model [26] is made of a spring and a dashpot in series (Figure 1), which is perfectly elastic and viscous. Since the deformation process is assumed to be quasistatic, inertia can be neglected and the force or stress is the same in both parts. The total deformation is the sum deformations of both parts. If the displacement of spring or dashpot is $\vec{u}^{s}$ or $\vec{u}^{d}$, the total displacement $\vec{u}$ is

$$
\vec{u}=\vec{u}^{s}+\vec{u}^{d}
$$

If $E$ is Young's modulus of the spring and $\eta$ is the viscosity of the dashpot, $\sigma_{s}$ and $\sigma_{d}$ are the stresses of the spring and the dashpot. The stresses are

$$
\sigma_{s}=E \vec{u}^{s}, \quad \sigma_{d}=\eta \dot{\vec{u}}^{d}, \quad \sigma_{s}=\sigma_{d}
$$

Given that the force on the spring and the depot is equal at any given time, the two parts can be processed independently.

2.2. Reference Frame. Two kinds of reference frames are used to describe deformations in a floating image that is deformed to a target image. One is the Lagrangian reference frame, which describes the deformations by observing changes in the positions and velocities of definite particles. The other is the Eulerian reference frame, which describes the deformations by observing velocity changes at fixed points. The Eulerian reference frame is suitable for large deformations because it does not trace the motion of the particles [27]. Therefore, the Eulerian reference frame is used to track the deformations in our method. Voxel grids are used as the fixed points. A particle at grid position $\vec{x}$ in floating image $I_{1}(\vec{x})$ at time $t$ is originated at the position $\vec{x}(t)-\vec{u}(\vec{x}, t)$, where $\vec{u}(\vec{x}, t)$ is the displacement field. The corresponding velocity field $\vec{v}(\vec{x}, t)$ is expressed as

$$
\vec{v}(\vec{x}, t)=\frac{\partial \vec{u}(\vec{x}, t)}{\partial t}+\sum_{j=1}^{3} v_{j} \frac{\partial \vec{u}(\vec{x}, t)}{\partial x_{j}}
$$

where $\vec{v}=\left[v_{1}, v_{2}, v_{3}\right]$. It comes from the derivative of the displacement field about time. The second term in (3) represents the nonlinearities of the displacement field.

2.3. The Viscoelastic Fluid Algorithm. We extend the Maxwell model to three dimensions. The spring becomes an elastic solid, and the dashpot becomes a viscous fluid. Therefore, the total deformation is similar to that in (1), where $\vec{u}^{s}=$ $\left[u_{1}^{s}, u_{2}^{s}, u_{3}^{s}\right]$ and $\vec{u}^{d}=\left[u_{1}^{d}, u_{2}^{d}, u_{3}^{d}\right]$ are displacements of the elastic solid part and the viscous fluid part, respectively. The force of the two parts is equal and is expressed as

$$
\vec{f}^{s}=\vec{f}^{d}
$$

where $\vec{f}^{s}$ and $\vec{f}^{d}$ are the forces acting on the elastic solid part and the viscous fluid part, respectively.

We used the continuum mechanics method to compute the displacements. The elastic solid displacements are described by the following partial differential equations:

$$
\mu^{s} \nabla^{2} \vec{u}^{s}+\left(\lambda^{s}+\mu^{s}\right) \nabla\left(\nabla \cdot \vec{u}^{s}\right)+\vec{f}^{s}=0,
$$

where $\mu^{s}$ and $\lambda^{s}$ are Lame's elastic coefficients and $\vec{u}^{s}=$ $\left[u_{1}^{s}(x, t), u_{2}^{s}(x, t), u_{3}^{s}(x, t)\right]$. The velocity of the viscous fluid part is determined using the following equation:

$$
\mu^{d} \nabla^{2} \vec{v}^{d}+\left(\lambda^{d}+\mu^{d}\right) \nabla\left(\nabla \cdot \vec{v}^{d}\right)+\vec{f}^{d}=0,
$$

where $\vec{v}^{d}=\left[v_{1}^{d}(x, t), v_{2}^{d}(x, t), v_{3}^{d}(x, t)\right]$ and $\mu^{d}$ and $\lambda^{d}$ are the viscosity constants. 
The velocity field of viscous fluid in an Eulerian reference frame can be determined by the following equation:

$$
\vec{v}^{d}(\vec{x}, t)=\frac{\partial \vec{u}^{d}(\vec{x}, t)}{\partial t}+\sum_{j=1}^{3} v_{j}^{d} \frac{\partial \vec{u}^{d}(\vec{x}, t)}{\partial x_{j}}
$$

The displacement fields $\vec{u}^{d}$ are updated iteratively over time step $\Delta t$ and are determined as follows:

$$
\vec{u}^{d}(t+\Delta t)=\vec{u}^{d}(t)+\Delta t\left[\vec{v}^{d}(t)-\nabla^{d}(t) \vec{v}^{d}(t)\right] .
$$

Time step $\Delta t$ is chosen according to the perturbation of the deformation field; we have

$$
\Delta t=\max \left(\left\|\vec{v}^{d}(\vec{x}, t)-\sum_{i=1}^{3} v_{i}^{d}(\vec{x}, t)\left[\frac{\partial \vec{u}(\vec{x}, t)}{\partial x_{i}}\right]\right\|\right) .
$$

The boundary conditions $\vec{u}^{s}(\vec{x}, t)=0$ and $\vec{v}^{d}(\vec{x}, t)=0$, and the total displacements on the boundary are set to zero. The elastic equation (5) and fluid equation (6) are solved simultaneously to obtain the total deformation.

2.4. The Adaptive Force. The motivation of the adaptive force is to speed up the registration. In the proposed method, the key parts of the partial differential equations (PDEs) (5) and (6) are the forces that drive the floating image to deform to the target image $I_{2}(\vec{x})$. The gradient of the SSD metrics is used as these forces. The standard force is defined as

$$
f=-\left.\alpha\left(I_{1}(\vec{x}-\vec{u}(\vec{x}, t))-I_{2}(\vec{x})\right) \nabla I_{1}(\vec{x})\right|_{\vec{x}-\vec{u}(\vec{x}, t)},
$$

where $\alpha$ is a constant.

The $\left.\nabla I_{1}(\vec{x})\right|_{\vec{x}-\vec{u}(\vec{x}, t)}$ is the gradient of the floating image at $\vec{x}-\vec{u}(\vec{x}, t) . I_{1}(\vec{x}-\vec{u}(\vec{x}, t))-I_{2}(\vec{x})$ is the difference in intensity between the deformed floating image and the target image and weighs the $\left.\nabla I_{1}(\vec{x})\right|_{\vec{x}-\vec{u}(\vec{x}, t)}$. The force is minimized at the location where the floating image and the target image are aligned.

As the registration progresses, the forces become smaller and the corresponding velocities also become smaller which lead to very small deformation in the iteration. Therefore, more iterations are needed to reach the final deformations. To speed up the registration, the forces should increase in the next iteration. Hence, an adaptive force is introduced to solve the problem in the proposed method. The maximum of the displacements should not stay below a specific threshold. When the maximum of the displacements is below the threshold at the current iteration, the forces are adjusted automatically to increase the maximum of the displacements in the next iteration. In our method, an empirical formula is used to define the adaptive force. The adaptive force in the Eulerian reference frame is expressed as

$$
\begin{aligned}
\vec{f}_{n+1} & =\vec{f}_{n+1}^{s}(\vec{x}, \vec{u}(\vec{x}, t))=\vec{f}_{n+1}^{d}(\vec{x}, \vec{u}(\vec{x}, t)) \\
& =-\alpha_{n+1} \times\left.\left(I_{1}(\vec{x}-\vec{u}(\vec{x}, t))-I_{2}(\vec{x})\right) \nabla I_{1}(\vec{x})\right|_{\vec{x}-\vec{u}(\vec{x}, t)},
\end{aligned}
$$

where $n+1$ is the next iteration and $\alpha_{n+1}$ is the function with respect to the maximum displacements of the current iteration. It is described as

$$
\alpha_{n+1}= \begin{cases}\alpha_{n}(1+\beta(\gamma-\max (\|\vec{u}(\vec{x}, t)\|))), & \text { if }(\max (\|\vec{u}(\vec{x}, t)\|))<\gamma, \\ \alpha_{n}, & \text { if }(\max (\|\vec{u}(\vec{x}, t)\|)) \geq \gamma,\end{cases}
$$

where $\max (\|\vec{u}(\vec{x}, t)\|)$ is the maximum of the displacements of the current iteration.

If the maximum displacement is below the threshold $\gamma$, $1+\beta(\gamma-\max (\|\vec{u}(\vec{x}, t)\|))$ should be larger than one, thereby making $\alpha_{n+1}$ larger than $\alpha_{n}$. The parameter $\alpha_{n+1}$ of the next iteration increases automatically. Therefore, the corresponding forces $\vec{f}_{n+1}$ also increase to prevent the displacement from becoming too small.

2.5. Implementation. When the floating image is deformed by the corresponding deformation field in the registration, the topology of the floating image should be preserved. Keeping all Jacobian of the deformation fields positive can preserve the topology. In the implementation, when the minimum of the Jacobian is below 0.5 , the transformation is applied to the floating image to produce a new image and the displacement field $\vec{u}$ is set to zero. The new image is then used as the floating image in the subsequent registration. The process continues as long as the SSD decreases. The pseudocode of the algorithm is as follows.

(1) Let $t=0$ and $\vec{u}(\vec{x}, 0)=0$.

(2) Calculate the force using (10), (11), and (12).

(3) If the SSD stops decreasing or the maximum number of iterations is reached, then stop.

(4) Solve PDEs (5) and (6) for displacements $\vec{u}^{s}(\vec{x}, t)$ and instantaneous velocity $\vec{v}^{d}(\vec{x}, t)$, respectively.

(5) Choose time step $\Delta t$ according to (8) and calculate $\vec{u}^{d}(\vec{x}, t)$.

(6) Calculate the total displacement $\vec{u}(\vec{x}, t)=\vec{u}^{s}(\vec{x}, t)+$ $\vec{u}^{d}(\vec{x}, t)$.

(7) If the Jacobian of the transformation is less than 0.5 , a new floating image is constructed, and then go to Step 1. Otherwise, update the displacement field according to (4), then set $t=t+\Delta t$, and go to Step 2 .

As it has been proved that relaxation is currently the best method [20], we solve PDEs (6) and (7) by means of successive overrelaxation [28].

2.6. Evaluation. The performance of the proposed method is evaluated on the basis of three analyses. The first analysis uses the golden deformation field $\vec{T}$. We then compare the recovered deformation field $\vec{T}^{\prime}$ by the root mean square (RMS) error over all voxels:

$$
\mathrm{RMS}=\sqrt{\frac{1}{N} \sum\left(\vec{T}^{\prime}(\vec{x})-\vec{T}(\vec{x})\right)^{2}},
$$

where $N$ is the number of total voxels in the image. 
TABLE 1: Computing time of various methods.

\begin{tabular}{lcccc}
\hline & FMSF (s) & FMAF (s) & MMSF (s) & MMAF (s) \\
\hline 2D simulated data & 36 & 12 & 26 & 18 \\
2D MRI data & 60 & 23 & 41 & 32 \\
IBSR database & 2891 & 1143 & 2025 & 1793 \\
Real data & 1256 & 532 & 1077 & 625 \\
\hline
\end{tabular}

The second analysis uses the mean of the SSD. They are defined as

$$
\mathrm{SSD}=\frac{1}{N} \sqrt{\sum\left(I_{2}(\vec{x})-T\left(I_{1}(\vec{x})\right)\right)^{2}},
$$

where $N$ is the number of total voxels in the image, $I_{1}(\vec{x})$ and $I_{2}(\vec{x})$ are floating and target images, respectively. While $T\left(I_{1}(\vec{x})\right)$ is the deformed floating image.

The last analysis uses tissue overlaps, which are defined as

$$
O=2 \times \frac{V\left(I_{2}(\vec{x}) \cap T\left(I_{1}(\vec{x})\right)\right)}{V\left(I_{2}(\vec{x})\right)+V\left(I_{1}(\vec{x})\right)}
$$

where $V$ is the volume of the tissues.

If the floating image completely matches the target image, the $O$ value would be one, and the RMS or SSD would be minimized. If there is no overlap between the two images, the $O$ value would be zero, and the RMS or SSD would be maximized.

\section{Experiments and Results}

Four experiments are conducted to demonstrate the proposed method. The first two experiments are about 2D data and the rest $3 \mathrm{D}$ volumes. The method is implemented in $\mathrm{C}$ and complies with $\mathrm{VC}++$ [29]. The whole image is modeled using a single set of material parameters for simplification purposes. The parameters $\mu^{s}, \lambda^{d}, \mu^{d}$ are all set to one and $\lambda^{s}$ is set to zero. The parameters $\alpha_{0}$ and $\beta$ are both set to 1 , and $\gamma$ is set to 0.8 voxels. The maximum iteration is set to 200 . These parameters are used in all the experiments.

3.1. $2 D$ Synthetic Datasets. The experiment shows that the proposed method can deal with large deformation well. The image sizes are $128 \times 128$ pixels, as shown in Figure 2 . Figure 2(a) is the floating image, with a rectangular image, and Figure 2(b) is the target image, a C-shape image. The results of FMSF, MMSF, FMAF, and MMAF are all successful to deform the rectangular image to $\mathrm{C}$-shape image. Figure 2(c) shows the results of FMAF. The computing costs are listed in the second row of Table 1 . The computing times of FMSF, FMAF, MMSF, and MMAF are 36, 12, 26, and 18 seconds, respectively. The ranking of speed from the fastest to the slowest is as follows: FMAF, MMAF, MMSF, and FMSF.

3.2. $2 D$ Brain MRI Datasets. The second experiment shows the effectiveness of the proposed method when it is applied to brain MRI. The floating image size is $256 \times 256$, as shown in Figure 3(a). This image is registered to a selected image by
TABLE 2: Comparison of RMS and SSD for various methods.

\begin{tabular}{lcccc}
\hline & FMSF & FMAF & MMSF & MMAF \\
\hline RMS (mm) & 0.3783 & 0.3066 & 0.2742 & 0.2412 \\
SSD & 0.0381 & 0.0293 & 0.0367 & 0.0293 \\
\hline
\end{tabular}

the finite element method [30] and obtains the deformation fields $\vec{T}$, which is used as the golden standard. The known deformation fields $\vec{T}$ are applied to the floating image to obtain a target image, as shown in Figure 3(b). The known field is shown in Figure 3(c) using the following equation:

$$
T=\sqrt{T_{x}^{2}+T_{y}^{2}}
$$

where $T_{x}, T_{y}$ are the known deformation fields in the $x$ and $y$ directions, respectively.

The fluid model and the Maxwell model with the standard forces and the adaptive forces are applied to the images. The computing time is listed in the third row of Table 1. The FMAD only costs 23 seconds, which is the fastest. By contrast, the MMAF and the MMSF cost 32 and 41 seconds, respectively, and they are the second and the third in terms of speed. The FMSF is the slowest and costs 60 seconds.

All of these methods can successfully deform the floating image to the target image. However, the matching accuracy is different. Table 2 lists the RMS and SSD acquired by the various methods. The RMS of FMSF, FMAF, MMSF, and MMFA are $0.3783,0.3066,0.2742$, and 0.2412 . According to RMS, MMAF has obtained the best result, followed by the MMSF, then FMAF, and FMSF last.

Figure 4 shows the difference among the known deformation fields obtained using various methods using the following equation:

$$
\Delta T=\sqrt{\left(T_{x}-T_{x}^{\prime}\right)^{2}+\left(T_{y}-T_{y}^{\prime}\right)^{2}},
$$

where $\Delta T$ is the difference of the known deformation fields. $T_{x}^{\prime}, T_{y}^{\prime}$ are the deformation fields of $x$ and $y$ directions, respectively. We find that regardless of which forces act, the differences of the known deformation fields with that of the fluid model (shown in Figures 4(a) and 4(b)) are much larger than that with the Maxwell model (shown in Figures 4(c) and 4(d)). The Maxwell model has obtained better results. Among them, the Maxwell model with the adaptive forces has obtained the best result, whereas the fluid model with the standard forces has the worst results. The SSD of FMSF, FMAF, MMSF, and MMFA are 0.0381, 0.0293, 0.0367, and 0.0293 . Based on the SSD, the results of the MMAF and FMAF have the same rank, whereas the results of the MMSF and the FMSF are ranked as third and fourth. It indicates that the adaptive force is superior to the standard force and the Maxwell model is more robust.

3.3. The Internet Brain Segmentation Repository (IBSR) Database. High-resolution 3D MR images are used to evaluate the proposed method. The MRI data are downloaded from the IBSR [31] and include 20 normal MR brain datasets and 


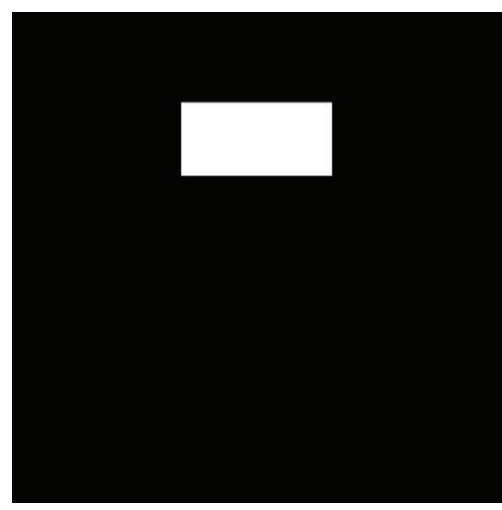

(a)

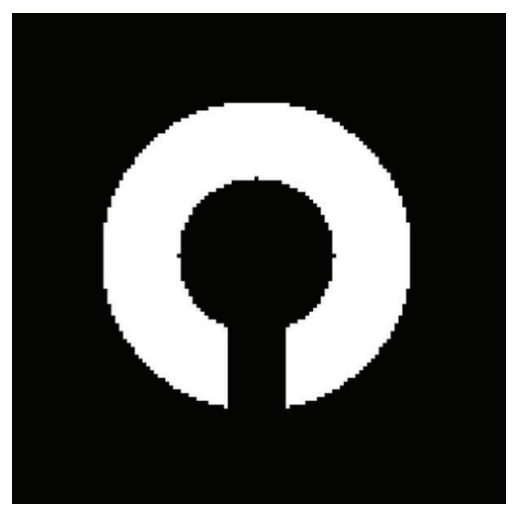

(b)

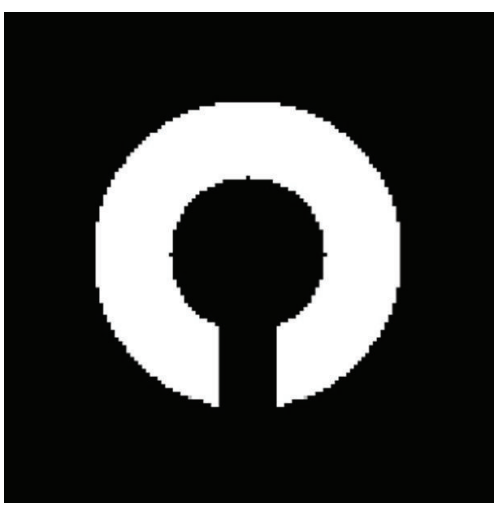

(c)

FIGURE 2: Results on 2D simulated datasets: (a) floating image, (b) target image, and (c) the result of Maxwell model with adaptive forces (MMAF).

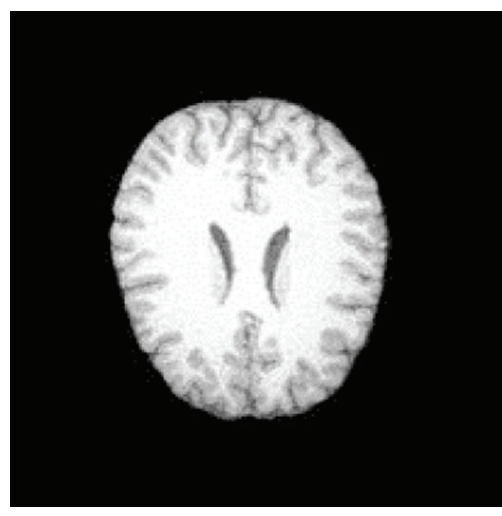

(a)

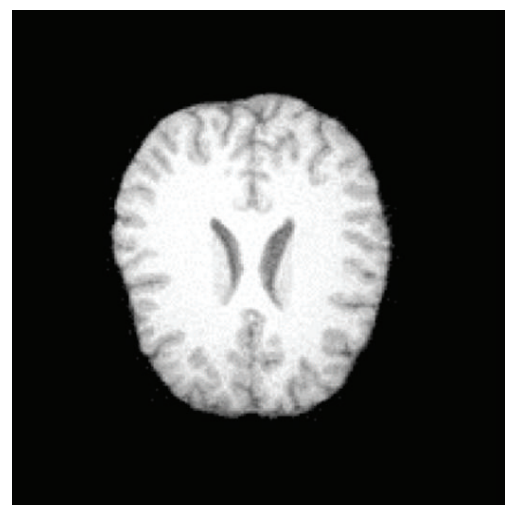

(b)

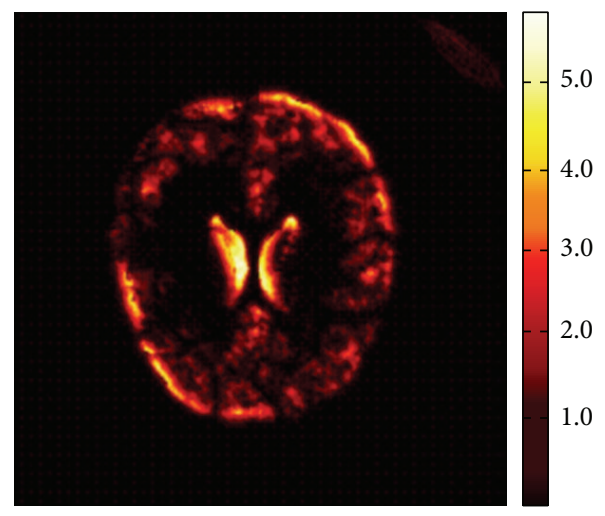

(c)

FIGURE 3: (a) Floating image. (b) Target image obtained by deforming the floating image with the known deformation field. (c) The known deformation field is obtained from FEM-based deformable registration method.

the skulls are all stripped. The column and row are 256 , and the slice is from 58 to 64 . The voxel size is $1 \times 1 \times 3 \mathrm{~mm}^{3}$. We have randomly selected 30 couples from the data to test the methods. The mean time is listed in the fourth row of Table 1. The computing times of FMSF, FMAF, MMSF, and MMAF are 2891, 1143, 2025, and 1793. The computation time of the fluid model with the adaptive forces is the fastest, the Maxwell model with the adaptive forces is the second, the model with the standard forces is the third, and the fluid model with the standard force is the slowest.

The mean of SSD is listed in the second row of Table 3. The SSD of the FMSF, FMAF, MMSF, and MMAF are 0.0553 , $0.0490,0.0504$, and 0.0484 , respectively. According to the SSD value, the results obtained by the MMAF and the FMAF are ranked as the first and the second, respectively, and those acquired by the MMSF are the third. The result obtained using the FMSF is the worst. However, finding the difference using visual inspections in the results is difficult. An example is shown in Figure 5.

The average tissue overlap values are listed in the second row of Table 4 . The overlap values of FMSF, FMAF, MMSF, and MMAF are $0.8813,0.8879,0.8872$, and 0.8917 . The overlap values of the Maxwell model are larger than that of the fluid model, and the model with the adaptive forces performs better than that with the standard forces.

3.4. Real Datasets. The real datasets are acquired from the local hospital. The scans are acquired using a SIEMENS TRIO 3 Tesla scanner installed at the Institute of Biophysics of the Chinese Academy of Sciences. These scans are T1 sagittal images $(\mathrm{TR}=1730 \mathrm{~ms}, \mathrm{TE}=3.93 \mathrm{~ms}$, thickness $=1.0 \mathrm{~mm}$, no gap, in-plane resolution $=256 \times 256$, slice $=192$, and flip angle $=15)$. The scans are resampled as $120 \times 120 \times 96 \mathrm{~mm}^{3}$ and the voxel size is $2.0 \times 2.0 \times 2.0 \mathrm{~mm}^{3}$. Thirty couples are randomly selected from the datasets. The mean time is listed in the fifth row of Table 1, and the SSD is listed in the third row of Table 3. The computing times of FMSF, FMAF, MMSF, and MMAF are $1256,532,1077$, and 625 , respectively. The SSD of FMSF, FMAF, MMSF, and MMAF are 0.0328, 0.0293, 0.0301, and 0.0279 . The computation costs and matching accuracy of the real datasets are similar to those of the IBSR datasets. The overlap values are listed in the third row of Table 4. The overlap values of FMSF, FMAF, MMSF, and MMAF are 


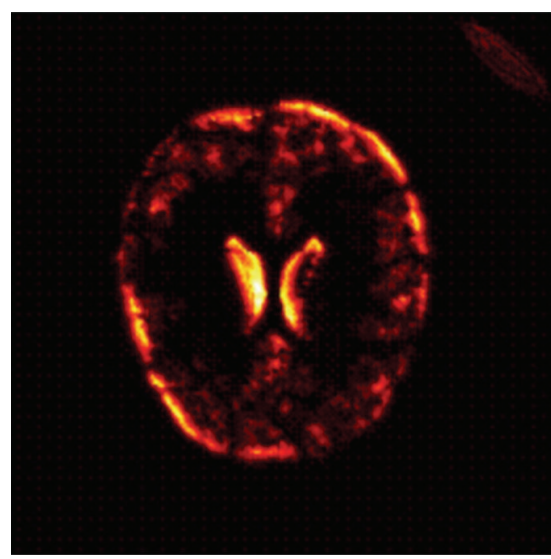

(a)

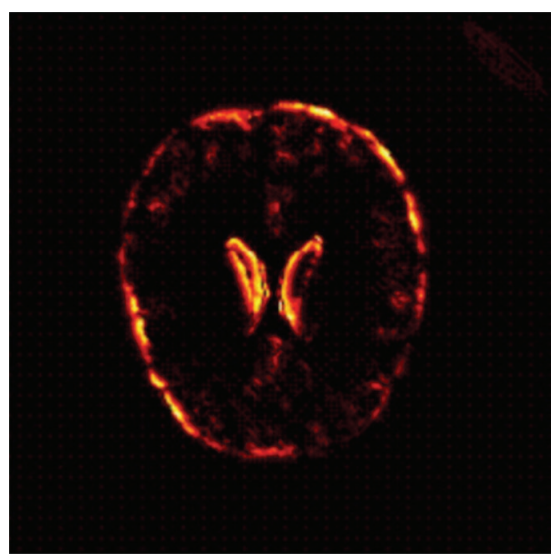

(c)

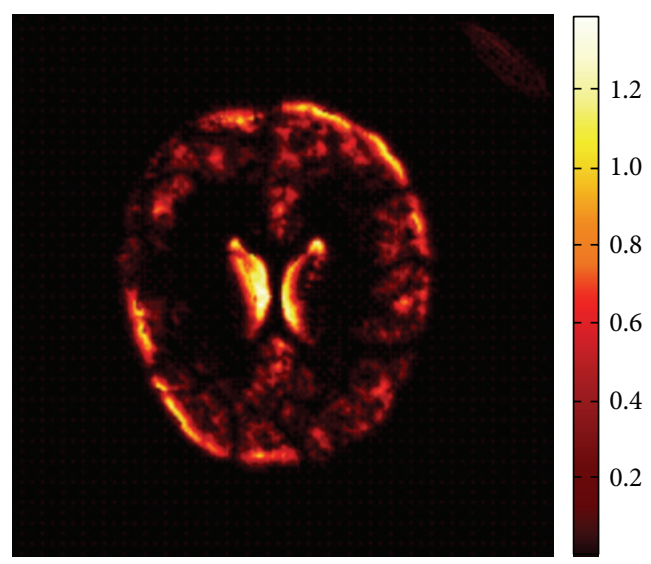

(b)

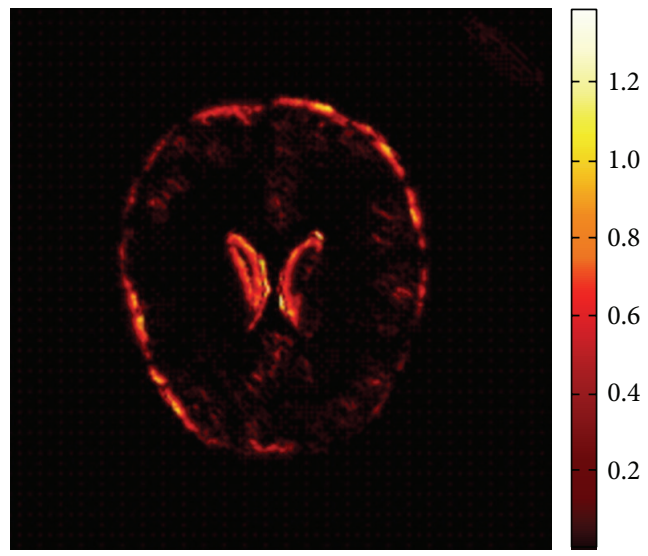

(d)

FIGURE 4: The differences of the known deformation fields with those got by various methods. (a) The difference of known deformation field with that obtained by the FMSF. (b) The difference of known deformation field with that obtained by the FMAF. (c) The difference of known deformation field with that obtained by the MMSF. (d) The difference of known deformation field with that obtained by the MMSF.

TABLE 3: Comparison of the mean values of SSD for various databases.

\begin{tabular}{lcccc}
\hline & FMSF & FMAF & MMSF & MMAF \\
\hline IBSR database & 0.0553 & 0.0490 & 0.0504 & 0.0484 \\
Real data & 0.0328 & 0.0293 & 0.0301 & 0.0279 \\
\hline
\end{tabular}

TABLE 4: Comparison of the overlap values for various methods.

\begin{tabular}{lccccc}
\hline & FMMI & FMSF & FMAF & MMSF & MMAF \\
\hline IBSR database & 0.8794 & 0.8813 & 0.8879 & 0.8872 & 0.8917 \\
Real data & 0.8801 & 0.8823 & 0.8912 & 0.8892 & 0.8920 \\
\hline
\end{tabular}

$0.8823,0.8912,0.8892$, and 0.8920 . The results are similar to those obtained in Section 3.3. The proposed method is also compared with a method using fluid model and mutual information (FMMI) [32]. As the second column of Table 4 shows, the overlaps of the IBSR dataset and the real dataset from FMMI are the smallest, respectively. This indicates that the SSD is better than mutual information in monomodal images.

\section{Conclusions}

The proposed method is driven by the fluid and elastic models [15-17], which uses the Maxwell model, a linear viscoelastic model that combines the properties of elastic and fluid models, to represent the image deformation. The proposed method introduces an adaptive force to speed up the registration.

The performances of the elastic and fluid models are compared in [17]. Therefore, we only compare the proposed method with the fluid method in this paper. The successive over relaxation method is used to solve the corresponding PDE, which is not the fastest but the most accurate among the evaluated methods. The computational cost can be reduced if PDEs are solved quickly, such as when using filter convolution [19] with a small filter width and parallel computing. However, the relative computation costs should be the same as those obtained in this paper. Actually, the fluid model is a special case of the linear viscoelastic model. When $\mu^{s}=0$ and $\lambda^{s}=0$, the linear viscoelastic model becomes a fluid model.

Our experimental results show that the linear viscoelastic model has several potential applications and that adaptive force can greatly reduce the registration time. The proposed 


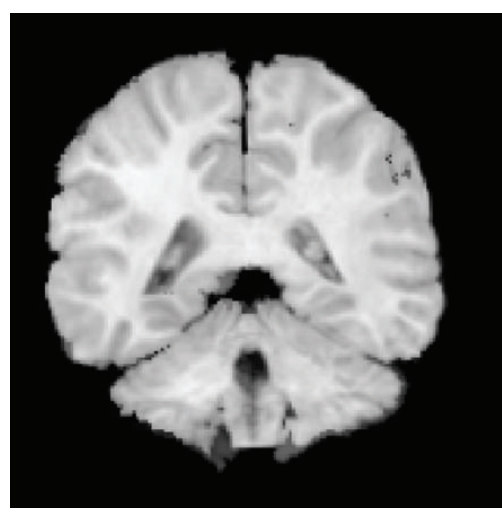

(a)

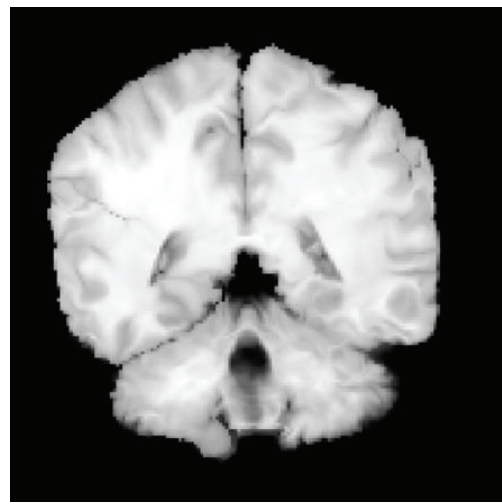

(d)

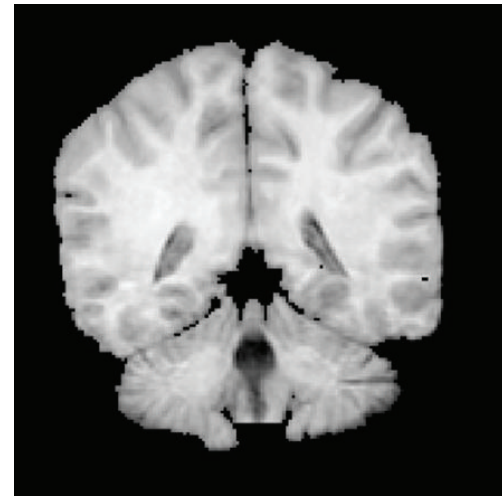

(b)

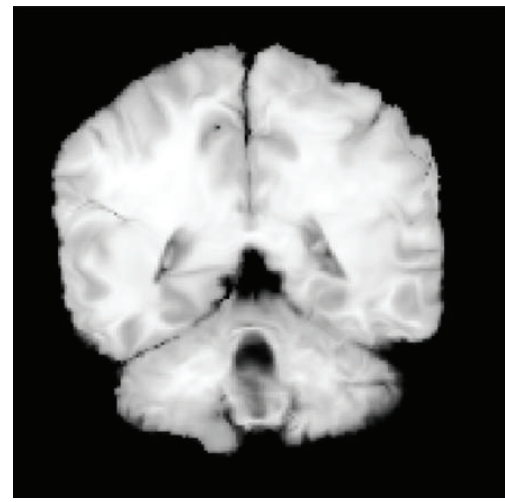

(e)

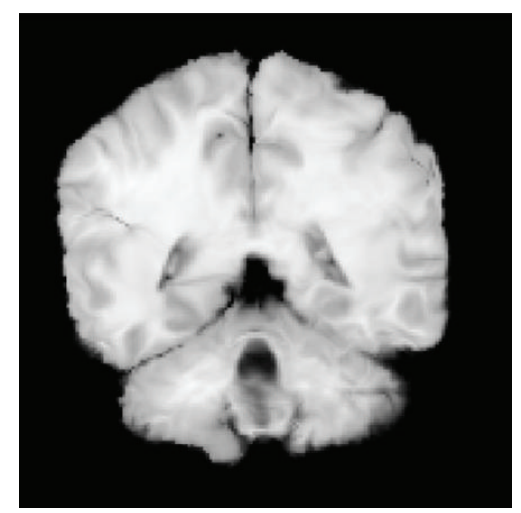

(c)

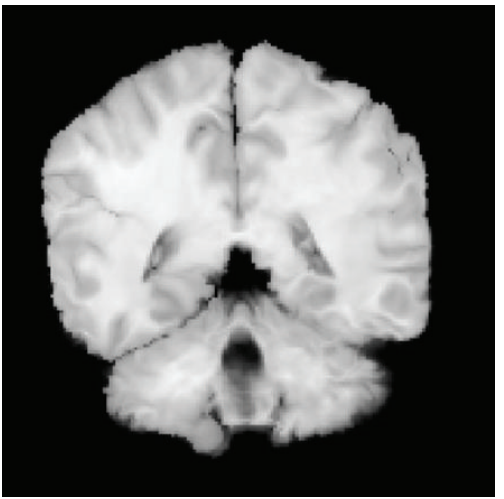

(f)

FIgURE 5: An example of the results of the 24th slice with various methods on IBSR Database is shown. (a) Floating image. (b) Target image. (c) The result of the fluid model with standard force (FMSF). (d) The result of the fluid model with adaptive force (FMAF). (e) The result of the Maxwell model with standard force (MMSF). (f) The result of the Maxwell model with adaptive force (MMAF).

method includes many parameters, and these should be analyzed further. We would also like to analyze the characterization of the transformation and how to obtain the optimal parameters for the corresponding transformation in the future.

\section{Conflict of Interests}

The authors declare that they have no conflict of interests.

\section{Acknowledgments}

This research was supported by the National Basic Research Program of China (2010CB732505 and 2013CB328806), the Key Projects in the National Science \& Technology Pillar Program (2013BAI01B01), the National Hi-Tech Research and Development Program (2013AA013703), and the National Natural Science Foundation of China (61272360).

\section{References}

[1] P. Aljabar, R. A. Heckemann, A. Hammers, J. V. Hajnal, and D. Rueckert, "Multi-atlas based segmentation of brain images: atlas selection and its effect on accuracy," NeuroImage, vol. 46, no. 3 , pp. $726-738,2009$.
[2] S. Joshi, B. Davis, M. Jomier, and G. Gerig, "Unbiased diffeomorphic atlas construction for computational anatomy," NeuroImage, vol. 23, pp. 151-160, 2004.

[3] S. Tang, Y. Fan, G. Wu, M. Kim, and D. Shen, "RABBIT: rapid alignment of brains by building intermediate templates," NeuroImage, vol. 47, no. 4, pp. 1277-1287, 2009.

[4] J. Burdett, J. Stevens, D. Flügel, E. Williams, J. S. Duncan, and L. Lemieux, "Increased sensitivity to pathological brain changes using co-registration of magnetic resonance imaging scans," Acta Radiologica, vol. 47, no. 10, pp. 1067-1072, 2006.

[5] D. Rueckert and J. A. Schnabel, "Medical image registration," in Biomedical Image Processing, pp. 131-154, Springer, Berlin, Germany, 2011.

[6] H. Chui and A. Rangarajan, "A new point matching algorithm for non-rigid registration," Computer Vision and Image Understanding, vol. 89, no. 2-3, pp. 114-141, 2003.

[7] A. Klein, S. S. Ghosh, B. Avants et al., "Evaluation of volumebased and surface-based brain image registration methods," NeuroImage, vol. 51, no. 1, pp. 214-220, 2010.

[8] P. Wen, "Medical image registration based-on points, contour and curves," in Proceedings of the International Conference on BioMedical Engineering and Informatics (BMEI '08), pp. 132136, May 2008.

[9] F. Maes, D. Vandermeulen, and P. Suetens, "Medical image registration using mutual information," Proceedings of the IEEE, vol. 91, no. 10, pp. 1699-1721, 2003. 
[10] M. Otte, "Elastic registration of fMRI data using Bezier-spline transformations," IEEE Transactions on Medical Imaging, vol. 20, no. 3, pp. 193-206, 2001.

[11] E. D’Agostino, F. Maes, D. Vandermeulen, and P. Suetens, "A viscous fluid model for multimodal non-rigid image registration using mutual information," Medical Image Analysis, vol. 7, no. 4, pp. 565-575, 2003.

[12] A. Klein, J. Andersson, B. A. Ardekani et al., "Evaluation of 14 nonlinear deformation algorithms applied to human brain MRI registration," NeuroImage, vol. 46, no. 3, pp. 786-802, 2009.

[13] J. Ashburner, "A fast diffeomorphic image registration algorithm," NeuroImage, vol. 38, no. 1, pp. 95-113, 2007.

[14] C. Broit, Optimal registration of deformed images [M.S. thesis], ProQuest, 1981.

[15] R. Bajcsy and S. Kovacic, "Multiresolution elastic matching," Computer Vision, Graphics, and Image Processing, vol. 46, pp. $1-21,1989$.

[16] M. Miller, G. Christensen, Y. Amit, and U. Grenander, "Mathematical textbook of deformable neuroanatomies," Proceedings of the National Academy of Sciences of the United States of America, vol. 90, no. 24, pp. 11944-11948, 1993.

[17] G. Christensen, R. Rabbitt, and M. Miller, "Deformable templates using large deformation kinematics," IEEE Transactions on Image Processing, vol. 5, no. 10, pp. 1435-1447, 1996.

[18] H. Lester and S. R. Arridge, "A survey of hierarchical non-linear medical image registration," Pattern Recognition, vol. 32, no. 1, pp. 129-149, 1999.

[19] M. Bro-Nielsen and C. Gramkow, "Fast fluid registration of medical images," in Visualization in Biomedical Computing, vol. 1131 of Lecture notes in computer science, pp. 267-276, Springer, Berlin, Germany, 1996.

[20] G. Wollny and F. Kruggel, "Computional cost of nonrigid registration algorithms based on fluid dynamics," IEEE Transactions on Medical Imaging, vol. 21, no. 8, pp. 946-952, 2002.

[21] B. Fischer and J. Modersitzki, "Fast diffusion registration," in Inverse problems, Image Analysis, and Medical Imaging: AMS Special Session on Interaction of Inverse Problems and Image Analysis, vol. 3, pp. 117-127, American Mathematical Society, New Orleans, La, USA, 2002.

[22] J. Modersitzki, Numerical Methods for Image Registration, Oxford University Press, New York, NY, USA, 2004.

[23] P. Cachier, E. Bardinet, D. Dormont, X. Pennec, and N. Ayache, "Iconic feature based nonrigid registration: the PASHA algorithm," Computer Vision and Image Understanding, vol. 89, no. 2-3, pp. 272-298, 2003.

[24] M. Hrapko, J. A. W. van Dommelen, G. W. M. Peters, and J. S. H. M. Wismans, "The mechanical behaviour of brain tissue: large strain response and constitutive modelling," Biorheology, vol. 43, no. 5, pp. 623-636, 2006.

[25] M. Shafieian and K. Darvish, "Viscoelastic properties of brain tissue under high-rate large deformation," in Proceedings of the ASME International Mechanical Engineering Congress and Exposition (IMECE '09), pp. 83-85, November 2009.

[26] R. Lakes, Viscoelastic Solids, CRC Press, New York, NY, USA, 1999.

[27] K. A. Saddi, C. Chefd'Hotel, and F. Cheriet, "Large deformation registration of contrast-enhanced images with volumepreserving constraint," in Medical Imaging 2007: Image Processing, Proceedings of SPIE, February 2007, 651203.

[28] W. H. Press, Numerical Recipes, Cambridge University Press, Cambridge, UK, 3rd edition, The Art of Scientific Computing, 2007.
[29] B. Zaratian, Microsoft Visual C++ 6.0 Programmer's Guide, Microsoft Press, 1998.

[30] L. Ibanez, W. Schroeder, L. Ng, and J. Cates, The ITK Software Guide, Citeseer, 2005.

[31] http://www.cma.mgh.harvard.edu/ibsr/.

[32] X. Huang, Nonrigid Image Registration Problem using Fluid Dynamics and Mutual Information, University of Connecticut, Storrs, Conn, USA, 2011. 


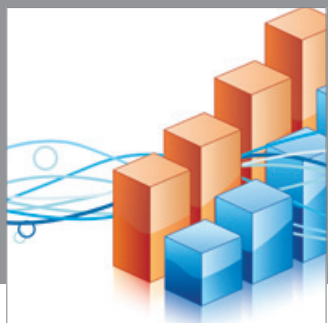

Advances in

Operations Research

mansans

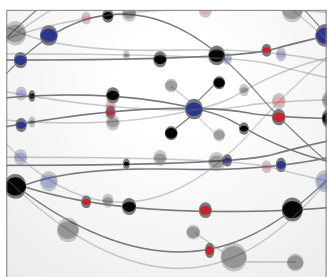

The Scientific World Journal
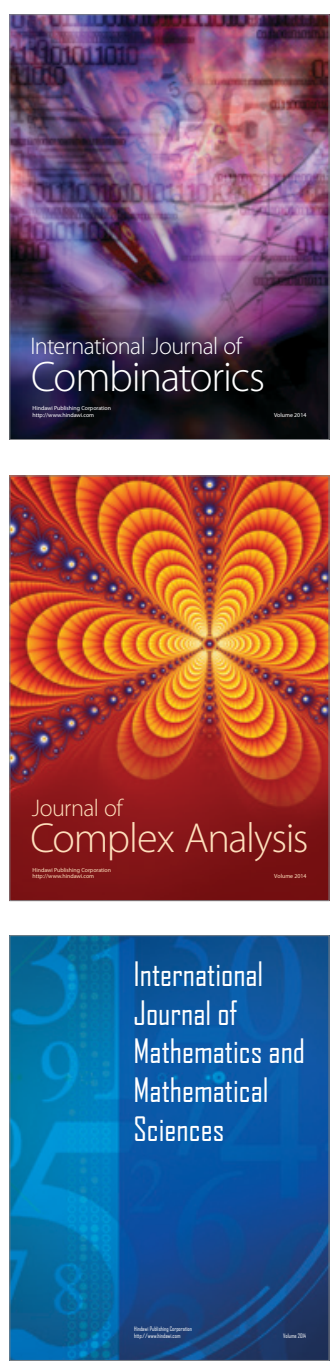
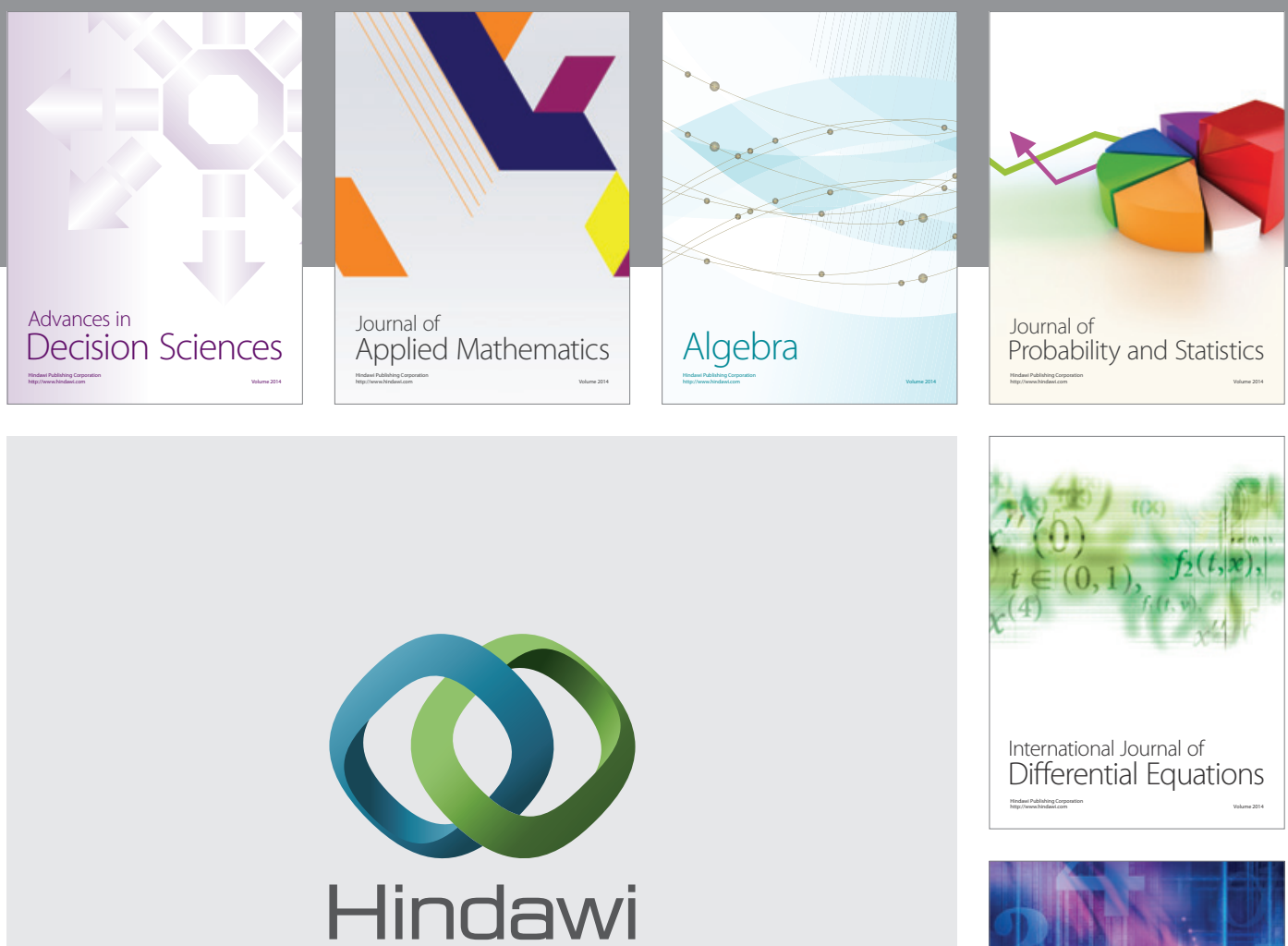

Submit your manuscripts at http://www.hindawi.com
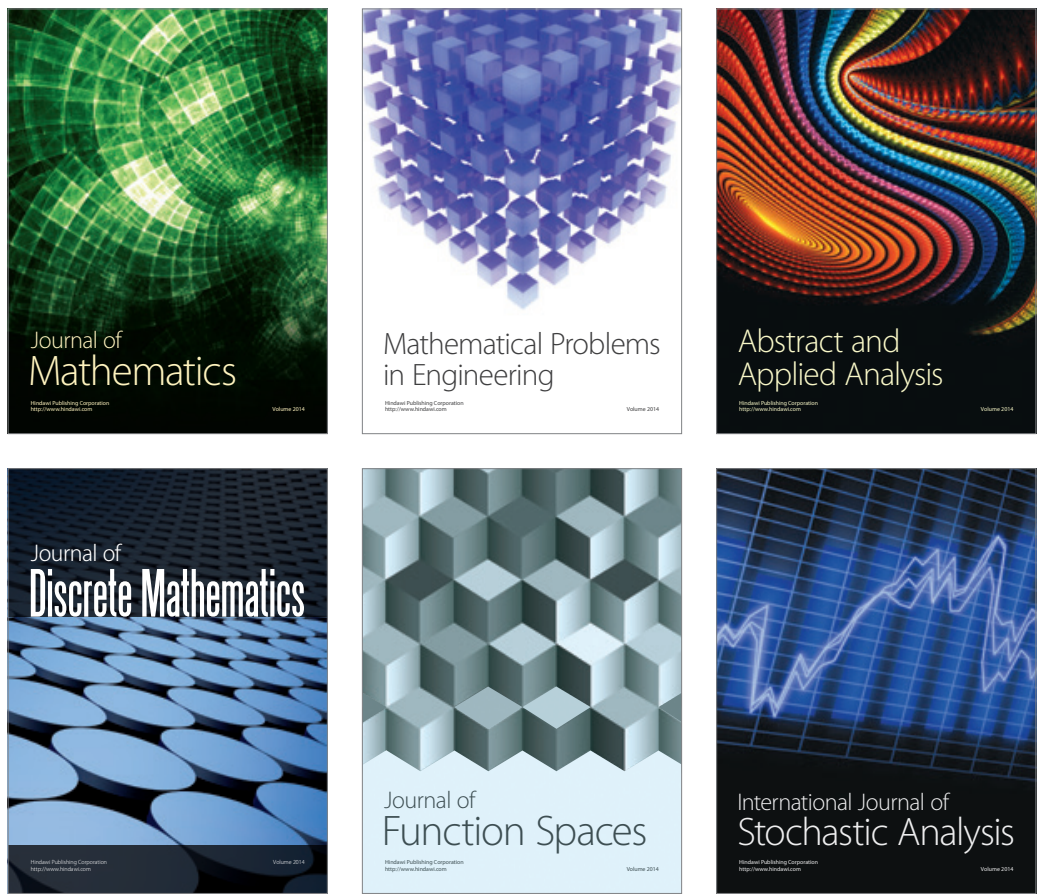

Journal of

Function Spaces

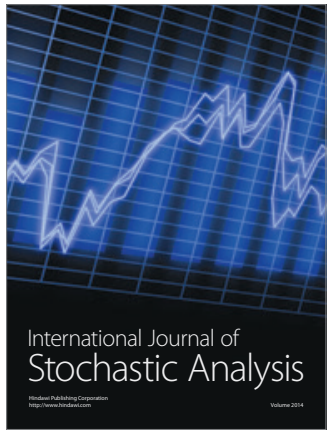

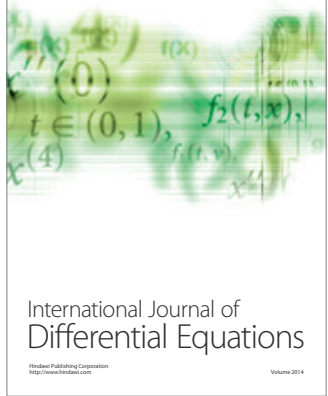
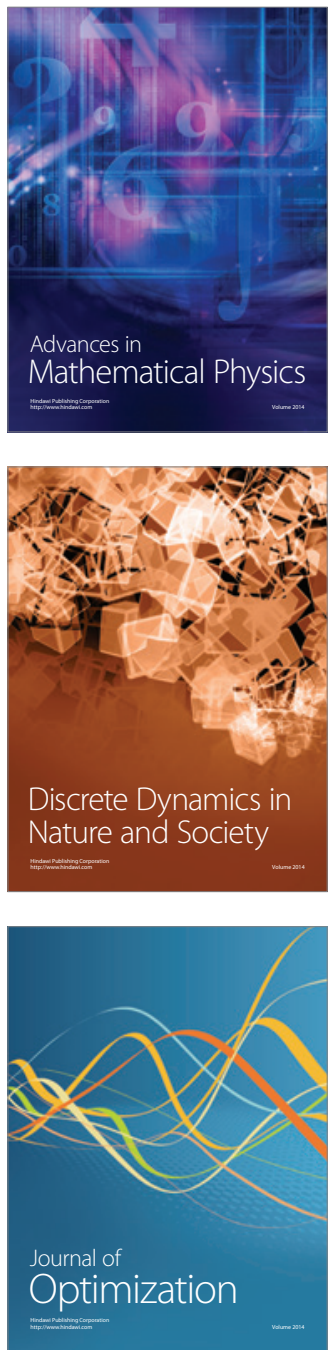